\title{
Can the current products of animal origin win the challenge with the offer of new foods?
}

\author{
Luigi Zicarelli* \\ Dipartimento di Medicina veterinaria e Produzioni Animali Università Federico II Napoli, Italy
}

The modern breeding has so far aimed to reduce the cost of food of animal origin to allow consumption even by the poor. The concept of quality did not take into account the sui generis taste but the longest possible shelf life, a fundamental requirement for large retailers. Even "fresh" cheeses can have a commercial life of up to 30 days when "freshness" is a distant memory.

Paradoxically, the rules in the EU have been dictated by northern Europeans who cannot teach anything in the food sector, because they consider the economic factor above all. In fact, the areas that gave rise to "typical" productions had to request exemptions to adapt the production to the rules of those who often "copied" many products.

The salami, for example, was created using only salt thanks to the meat of Mediterranean pigs, characterized by an excellent fat / muscle ratio ideal for their conservation. The Lucan women, as slaves, introduced the "lucaniche" to Rome, which in Brazil are called calabreza because the technology was introduced by Calabrian emigrants. Long ago, the Etruscans and Spaniards created ham using black Mediterranean pigs. The ham was particularly appreciated by the Romans so much that in Rome existed via Panisperma (bread and ham).

After the Second World War, the Anglo-Danish genetics gave birth to subjects characterized by remarkable growth and endowed with muscular masses suitable for direct consumption or transformation into wurstel, but not suitable for processing into typical salamis for which, with the exception of DOP products, today it is necessary to use nitrates. This genetics has greatly reduced family production, which traditionally uses only salt that does not allow the preservation of this type of meat. From a dietary point of view they are better meats, but they don't remember anything about the pig. The meat of these pork processed into cured meats provides less saturated fats that are harmful to the cardiovascular system, but for their preservation it is necessary to use nitrates that are considered carcinogenic. Unlike northern European countries, where the consumption of light pigs increases, in Italy, where food is also tradition and culture, increasingly heavier pigs have recently been requested. This turnaround in 2018 increased consumption by $4 \%$.

Currently, however, small farms are spreading in which the pigs are raised outdoors, have a higher post-weaning mortality and lower daily growths. Slower growth favors a concentration of flavors that meets the expectations of the most demanding consumers. Due to the characteristics of this type of breeding and to the greater well-being, to which the consumer today is particularly attentive, these pigs are particularly in demand and are conveniently remunerated.

The poor quality, in my opinion, of chicken meat has not prevented its consumption from increasing thanks to the convenient price and to the false news of Midia about its healthiness and its poor impact on the GW. This happened despite the fact that it is a species with a more rapid growth than that of a neoplastic mass (50 times in 50 days). The consumption of turkey meat is also growing, a species that, if released in the wild, would not be able to reproduce. These evidences do not worry the consumer even if recently the consumption of chickens with more solid muscle masses and with growths that require more than double the time necessary for the subjects bred with intensive techniques to reach the slaughter weight is increasing. Currently this type of chicken represents in Italy $1 \%$ of conventional production but in the last two years consumption has increased by $30 \%$, despite the higher costs.

The classification of cattle carcasses by the EU rewards those characterized by muscular hypertrophy similar to that obtained with anabolic steroids. It does not matter if the meat of these subjects no longer has the "sui generis" flavor, therefore it cannot be distinguished, if evaluated "blindly", from that of the chicken or the pig. The carcasses of a Rubia Gallega, a Chianina, a Nelore or a Podolico bred in the pasture are penalized. The carcass of an industrially bred "Bleu Belga" beef provides very popular tasteless meat. The omega6: omega 3 ratio of the industrial steak can reach the value of 30: 1 while that of the grazing animals has a ratio of 2: 1. Cattle fed as "ruminants" grow less but provide leaner meat which reduces cholesterol levels "Male" and LDL which is richer, three to five times, than CLA and vitamin E. Buffalo meat, regardless of rationing, provides lean meat. Finnish women who had received higher levels of CLA in their diet had a breast cancer risk $60 \%$ lower than those taking lower levels of CLA. The pastures in Italy are scarce but it is not impossible, in the final phase, to use diets with more forage to give the meat more favorable nutraceutical characteristics, exactly the opposite of what is happening today. There would be a slowdown in growth and higher costs, but a better product under the nutraceutical aspect.

Similar considerations apply to milk. Recently, thanks to an experimental plan devised by Infascelli, Lombardi and Mollica (2017, 2018), it has been shown that cow's milk fed with a forage / concentrate ratio: 70/30 favorably affects lipid metabolism in rats, the leptin ratio: adiponectin and reduces the amount of inflammatory substances and those responsible for oxidative stress and lipid accumulation in the liver $[1,2]$.

From these results it can be stated that the less productive breeds should be preferred, because they are more suitable to be rationed with a forage / concentrate ratio of 70/30 able to provide a nutraceutical milk

${ }^{*}$ Correspondence to: Luigi Zicarelli, Dipartimento di Medicina veterinaria e Produzioni Animali Università Federico II Napoli, Italy, Tel: 0812536059; E-mail: zicarell@unina.it

Received: May 13, 2019; Accepted: June 25, 2019; Published: June 28, 2019 
capable of putting an end to the demonization of products of origin animal. Recently, a series of functional molecules useful for human health has been highlighted in the milk and meat of ruminants, whose presence can be influenced by farming techniques. In particular, it has been shown that $\delta$-Valerobetaine $(\delta \mathrm{VB})$, present in buffalo milk, has a potential beneficial role and promises to be a new dietary compound with healthy properties $[3,4]$.

The sheep and goat products are certainly the best from the nutraceutical point of view. However, their consumption has gradually decreased due to incomplete information on the media that has always avoided reporting that the longest-lived populations are Italian ones in the inland areas of Sardinia and Cilento and Caucasus, which consume sheep and goat products.

Further cost reduction, production of functional foods or exaltation of flavors? The zootechnics of the future will have to work on the harmony between these needs, without underestimating that the increase in production coincides with tasteless tastes. The consumption of increasingly "tasteless" foods flavored with kechup is the premise for encouraging the consumption of worms, crickets and "Shmeat" meat, proposed, not surprisingly, by researchers from countries that have no taste and culinary traditions. The future problem of breeding in addition to the cost of the products will be their taste and the presence of nutraceutical substances. These needs are difficult to reconcile because the high productions, necessary to contain costs, correspond to insipid foods that require seasonings that mask the sui generis flavor and increasingly distance the consumer from traditional foods and bring them closer to new food sources.

\section{References}

1. Trinchese G, Cavaliere G, Penna E, Filippo CD, Cimmino F, et al. (2017) Milk from Cow Fed with High Forage/Concentrate Ratio Diet: Beneficial Effect on Rat Skeletal Muscle Inflammatory State and Oxidative Stress Through Modulation of Mitochondrial Functions and AMPK Activity. Front Physiol 9: 1969. [Crossref]

2. Servillo L, D'Onofrio N, Giovane A, Casale R, Cautela D, et al. (2018) Ruminan meat and milk contain d-valerobetaine, another precursor of trimethylamine $\mathrm{N}$-oxide (TMAO) like ?-butyrobetaine. Food Chemistry 260: 193-199.

3. Servillo L, D'Onofrio N, Giovane A, Casale R, Cautela D, et al. (2018) Ruminan meat and milk contain $\delta$-valerobetaine, another precursor of trimethylamine $\mathrm{N}$-oxide (TMAO) like $\gamma$-butyrobetaine. Food Chemistry 260: 193-199.

4. Servillo L, D'Onofrio N, Neglia G, Casale R, Cautela D (2018) Carnitine Precursors and Short-Chain Acylcarnitines in Water Buffalo Milk. J Agric Food Chem 66: 81428149 .

Copyright: (2019 Zicarelli L. This is an open-access article distributed under the terms of the Creative Commons Attribution License, which permits unrestricted use, distribution, and reproduction in any medium, provided the original author and source are credited. 